\title{
Author and Keyword Indexes for Volume 51
}

\section{AUTHOR INDEX TO VOLUME 51}

Aguilera, R.V., see Fainshmidt, S.

Aguinis, H., Ramani, R.S., Cascio, W.F., Methodological practices in international business research: An after-action review of challenges and solutions, 1606

Aguzzoli, R., see Nielsen, B.B.

Allen, L., Chakraborty, S., Hazarika, S., Su, C.-H., Bank dependence in emerging countries: Crossborder information percolation in mutual fund equity investing, 218

Ambos, T.C., Fuchs, S.H., Zimmermann, A., Managing interrelated tensions in headquarterssubsidiary relationships: The case of a multinational hybrid organization, 906

Arikan, I., see Li., C.

Auer-Rizzi, W., see Dastmalchian, A.

Backmann, J., Kanitz, R., Tian, A.W., Hoffmann, P., Hoegl, M., Cultural gap bridging in multinational teams, 1283

Bacon, N., see Dastmalchian, A.

Bayraktar, S., see Dastmalchian, A.

Beamish, P.W., see Sartor, M.A.

Benito, G.R.G., see Grøgaard, B.

Benito, G.R.G., see Iurkov, V.

Berry, H., Internationalizing firm innovations: The influence of multimarket overlap in knowledge activities, 963

Beugelsdijk, S., see Kostova, T.

Beugelsdijk, S., van Witteloostuijn, A., Meyer, K.E., A new approach to data access and research transparency (DART), 887

Birkinshaw, J., see Prashantham, S.

Blyton, P., see Dastmalchian, A.

Bodla, A.A., see Dastmalchian, A.

Brewster, C., Book Review, 146

Brouthers, L.E., see Marshall, V.B.

Buckley, P.J., China's belt and road initiative: Changing the rules of globalization, 279

Buckley, P.J., The theory and empirics of the structural reshaping of globalization, 1592

Buffardi, L.C., see Rockstuhl, T.
Caligiuri, P., De Cieri, H., Minbaeva, D., Verbeke, A., Zimmermann, A., International HRM insights for navigating the COVID-19 pandemic: Implications for future research and practice, 697

Cannizzaro, A.P., Social influence and MNE strategic response to political risk: A global network approach, 829

Cannon, J.N., Hu, B., Lee, J.J., Yang, D., The effect of international takeover laws on corporate resource adjustments: Market discipline and/or managerial myopia?, 1447

Cantwell, J., see Cuypers, I.R.P.

Cantwell, J., see Lundan, S.

Cascio, W.F., see Aguinis, H.

Castellani, D., Lavoratori, K., The lab and the plant: Offshore R\&D and co-location with production activities, 121

Chakraborty, S., see Allen, L.

Chapple, W., see Gutierrez-Huerter O.G.

Chidlow, A., see Nielsen, B.B.

Chua, C.H., see Kostova, T.

Coeurderoy, R., The new global road map: Enduring strategies in turbulent times, 693

Cotton, R., see Dastmalchian, A.

Couper, C., Reuber, A.R., Lost that lovin' feeling: The erosion of trust between small, high-distance partners, 326

Coviello, N., see Monaghan, S.

Craig, T., see Dastmalchian, A.

Cumming, D., Lopez-de-Silanes, F., McCahery, J.A., Schwienbacher, A., Tranching in the syndicated loan market around the world, 95

Cuypers, I.R.P., Ertug, G., Cantwell, J., Zaheer, A., Kilduff, M., Making connections: Social networks in international business, 714

Dası, À., see Pedersen, T.

Dastmalchian, A., Bacon, N., McNeil, N., Steinke, C., Blyton, P., Satish Kumar, M., Bayraktar, S., Auer-Rizzi, W., Bodla, A.A., Cotton, R., Craig, T., Ertenu, B., Habibi, M., Huang, H.J., İmer, H.P., Isa, C.R., Ismail, A., Jiang, Y., Kabasakal, H., Meo Colombo, C., Moghavvemi, S., Mukherjee, T., Musa, G.B., Sugai, P., Tang, N., Thang, T.T.N., 
Varnali, R., High-performance work systems and organizational performance across societal cultures, 353

De Cieri, H., see Caligiuri, P.

Den Hartog, D.N., see Watts, L.L.

Deng, P., Delios, A., Peng, M.W., A geographic relational perspective on the internationalization of emerging market firms, 50

Eden, L., Nielsen, B.B., Research methods in international business: The challenge of complexity, 1623

Eisenberger, R., see Rockstuhl, T.

Ertenu, B., see Dastmalchian, A.

Ertug, G., see Cuypers, I.R.P.

Fainshmidt, S., Witt, M.A., Aguilera, R.V., Verbeke, A., The contributions of qualitative comparative analysis (QCA) to international business research, 455

Fisch, J.H., Schmeisser, B., Phasing the operation mode of foreign subsidiaries: Reaping the benefits of multinationality through internal capital markets, 1223

Fleury, M.T.L., see Li, J.

Ford, M.T., see Rockstuhl, T.

Fortanier, F., Miao, G., Kolk, A., Pisani, N., Accounting for firm heterogeneity in global value chains, 432

Fuchs, S.H., see Ambos, T.C.

Gande, A., John, K., Nair, V.B., Senbet, L.W., Taxes, Institutions and Innovation: Theory and International Evidence, 1415

Gardner, E.C., see Nielsen, B.B.

Gaur, A., see Kumar, V.

Gold, S., see Gutierrez-Huerter O.G.

Gözübüyük, R., Kock, C.J., Ünal, M., Who appropriates centrality rents? The role of institutions in regulating social networks in the global Islamic finance industry, 764

Graafland, J., Noorderhaven, N., Culture and institutions: How economic freedom and long-term orientation interactively influence corporate social responsibility, 1034

Grøgaard, B., Rygh, A., Benito, G.R.G., Correction to: Bringing corporate governance into internalization theory:State ownership and foreign entry strategies, 1351

$\mathrm{Gu}$, Q., see Wang, S.L.

Gugler, P., see Rosa, B.
Gutierrez-Huerter O, G., Moon, J., Gold, S., Chapple, W., Micro-processes of translation in the transfer of practices from MNE headquarters to foreign subsidiaries: The role of subsidiary translators, 389

Habibi, M., see Dastmalchian, A.

Harhoff, P.-L., see Hutzschenreuter, T.

Hazarika, S., see Allen, L.

Hennart, J.-F., More than intent: A bundling model of MNE-SME interactions, 1176

Hirsch, P., see Wang, S.L.

Hitt, M.A., see $\mathrm{Xu}, \mathrm{K}$.

Hoegl, M., see Backmann, J.

Hoffmann, P., see Backmann, J.

Hoskisson, R.E., see Kim, H.

$\mathrm{Hu}, \mathrm{B}$. , see Cannon, J.N.

Huang, T., Wu, F., Yu, J., Zhang, B., Investor protection and the value impact of stock liquidity, 72

Huang, H.J., see Dastmalchian, A.

Hult, G.T.M., Gonzalez-Perez, M.A., The theoretical evolution and use of the Uppsala Model of internationalization in the international business ecosystem, 38

Hutzschenreuter, T., Harhoff, P.-L., National capital city location and subsidiary portfolio expansion: The negative effect of geographic distance to the capital city at inception on the speed of subsequent investments, 1107

İmer, H.P., see Dastmalchian, A.

Isa, C.R., see Dastmalchian, A.

Ismail, A., see Dastmalchian, A.

Iurkov, V., Benito, G.R.G., Change in domestic network centrality, uncertainty, and the foreign divestment decisions of firms, 788

Jeong, Y., Siegel, J.I., How important is regional vs. global scope? An examination of U.S. multinationals, 1142

Jiang, Y., see Dastmalchian, A.

Johanson, J., see Vahlne, J.-E.

John, K., see Gande, A.

Kabasakal, H., see Dastmalchian, A.

Kanagaretnam, K., Kong, X., Tsang, A., Home and foreign host country IFRS adoption and crossdelisting, 1008

Kang, H., see Seo, E.

Kanitz, R., see Backmann, J. 
Kano, L., Tsang, E.W.K., Yeung, H.W., Correction to: Global value chains: A review of the multidisciplinary literature, 1353

Kano, L., Tsang, E.W.K., Yeung, H.W., Global value chains: A review of the multi-disciplinary literature, 577

Karafyllia, M., see Nielsen, B.B.

Keig, D.L., see Marshall, V.B.

Khurshed, A., Mohamed, A., Schwienbacher, A., Wang, F., Do venture capital firms benefit from international syndicates?, 986

Kilduff, M., see Cuypers, I.R.P.

Kim, H., Wu, J., Schuler, D.A., Hoskisson, R.E., Chinese multinationals' fast internationalization: Financial performance advantage in one region, disadvantage in another, 1076

Kim, M., Lampert, C.M., Roy, R., Regionalization of R\&D activities: (Dis)economies of interdependence and inventive performance, 1054

Kock, C.J., see Gözübüyük, R.

Kohlhase, S., Pierk, J., The effect of a worldwide tax system on tax management of foreign subsidiaries, 1312

Kolk, A., see Fortanier, F.

Kolk, A., see van der Straaten, K.

Kong, X., see Kanagaretnam, K.

Kor, Y., see Tan, D.

Kostova, T., Beugelsdijk, S., Scott, W.R., Kunst, V.E., Chua, C.H., van Essen, M., The construct of institutional distance through the lens of different institutional perspectives: Review, analysis, and recommendations, 467

Kumar, V., Singh, D., Purkayastha, A., Popli, M., Gaur, A., Springboard internationalization by emerging market firms: Speed of first cross-border acquisition, 172

Kunst, V.E., see Kostova, T.

Kurtessis, J.N., see Rockstuhl, T.

la Cour, L., see McGaughey, S.L.

Lampert, C.M., see Kim, M.

Larraza-Kintana, M., see Shijaku, E.

Larsen, M.M., see Pedersen, T.

Lavoratori, K., see Castellani, D.

Lee, J.J., see Cannon, J.N.

Levine, R., Lin, C., Shen, B., Cross-border acquisitions: Do labor regulations affect acquirer returns?, 194

Levinthal, D.A., Learning in context: Grateful reflections on reflections, 1537

Lewin, A.Y., Massini, S., Peeters, C., Absorptive capacity, socially enabling mechanisms, and the role of learning from trial and error experiments: A tribute to Dan Levinthal's contribution to international business research, 1579

Li, C., see Meyer, K.E.

Li, D., see Liu, C.

Li, J., Fleury, M.T.L., Overcoming the liability of outsidership for emerging market MNEs: A capability-building perspective, 23

Li., C., Arikan, I., Shenkar, O., The impact of country-dyadic military conflicts on market reaction to cross-border acquisitions, 299

Lin, C., see Levine, R.

Lindner, T., Puck, J., Verbeke, A., Misconceptions about multicollinearity in international business research: Identification, consequences, and remedies, 283

Lissoni, F., see Useche, D.

Liu, C., Li, D., Divestment response to host-country terrorist attacks: Inter-firm influence and the role of temporal consistency, 1331

Lopez-de-Silanes, F., see Cumming, D.

Lorenzen, M., Mudambi, R., Schotter, A., International connectedness and local disconnectedness: MNE strategy, city-regions and disruption, 1199

Lundan, S., Cantwell, J., The local co-evolution of firms and governments in the Information Age, 1523

Luo, Y., Adaptive learning in international business, 1557

Mahoney, J.T., see Tan, D.

Marshall, V.B., Brouthers, L.E., Keig, D.L., RIMS: A new approach to measuring firm internationalization, 1133

Massini, S., see Lewin, A.Y.

McCahery, J.A., see Cumming, D.

McGaughey, S.L., Raimondos, P., la Cour, L., Foreign influence, control, and indirect ownership: Implications for productivity spillovers, 1392

McNeil, N., see Dastmalchian, A.

McWilliam, S.E., Nielsen, B.B., Global value chains and development: Redefining the contours of 21st century capitalism, 1347

Meo Colombo, C., see Dastmalchian, A.

Mesdaghinia, S., see Rockstuhl, T.

Meyer, K.E., Li, C., Schotter, A.P.J., Managing the MNE subsidiary: Advancing a multi-level and dynamic research agenda, 538

Meyer, K.E., see Beugelsdijk, S.

Miao, G., see Fortanier, F.

Miguelez, E., see Useche, D. 
Miller, S.R., see Nielsen, B.B.

Miller, S.R., see $\mathrm{Xu}, \mathrm{K}$.

Minbaeva, D., see Caligiuri, P.

Moghavvemi, S., see Dastmalchian, A.

Mohamed, A., see Khurshed, A.

Monaghan, S., Tippmann, E., Coviello, N., Born digitals: Thoughts on their internationalization and a research agenda, 11

Moon, J., see Gutierrez-Huerter O.G.

Mudambi, R., see Lorenzen, M.

Mukherjee, T., see Dastmalchian, A.

Musa, G.B., see Dastmalchian, A.

Nair, V.B., see Gande, A.

Nielsen, B.B., see Eden, L.

Nielsen, B.B., see McWilliam, S.E.

Nielsen, B.B., Welch, C., Chidlow, A., Miller, S.R., Aguzzoli, R., Gardner, E.C., Karafyllia, M., Pegoraro, D., Fifty years of methodological trends in JIBS: Why future IB research needs more Triangulation, 1483

Noorderhaven, N., see Graafland, J.

Papanastassiou, M., Pearce, R., Zanfei, A., Changing perspectives on the internationalization of $R \& D$ and innovation by multinational enterprises: A review of the literature, 623

Pearce, R., see Papanastassiou, M.

Pedersen, T., Larsen, M.M., Dası, À., Searching locally and globally: Applying Daniel Levinthal's scholarship to international business, 1541

Peeters, C., see Lewin, A.Y.

Pegoraro, D., see Nielsen, B.B.

Pierk, J., see Kohlhase, S.

Pisani, N., see Fortanier, F.

Pisani, N., see van der Straaten, K.

Popli, M., see Kumar, V.

Prashantham, S., Birkinshaw, J., MNE-SME cooperation: An integrative framework, 1161

Puck, J., see Lindner, T.

Purkayastha, A., see Kumar, V.

Raimondos, P., see McGaughey, S.L.

Ramani, R.S., see Aguinis, H.

Reuber, A.R., see Couper, C.

Rockstuhl, T., Eisenberger, R., Shore, L.M., Kurtessis, J.N., Ford, M.T., Buffardi, L.C., Mesdaghinia, S., Perceived organizational support (POS) across 54 nations: A cross-cultural meta-analysis of POS effects, 933
Rosa, B., Gugler, P., Verbeke, A., Regional and global strategies of MNEs: Revisiting Rugman \& Verbeke (2004), 1045

Roy, R., see Kim, M.

Rygh, A., see Grøgaard, B.

Sartor, M.A., Beamish, P.W., Integration-oriented strategies, host market corruption and the likelihood of foreign subsidiary exit from emerging markets, 414

Satish Kumar, M., see Dastmalchian, A.

Schaefer, K.J., Catching up by hiring: The case of Huawei, 1506

Schmeisser, B., see Fisch, J.H.

Schotter, A., see Lorenzen, M.

Schotter, A.P.J., see Meyer, K.E.

Schuler, D.A., see Kim, H.

Schwienbacher, A., see Cumming, D.

Schwienbacher, A., see Khurshed, A.

Scott, W.R., see Kostova, T.

Senbet, L.W., see Gande, A.

Seo, E., Kang, H., Song, J., Blending talents for innovation: Team composition for cross-border $\mathrm{R} \& \mathrm{D}$ collaboration within multinational corporations, 851

Shen, B., see Levine, R.

Shenkar, O., see Li., C.

Shijaku, E., Larraza-Kintana, M., Urtasun-Alonso, A., Network centrality and organizational aspirations: A behavioral interaction in the context of international strategic alliances, 813

Shore, L.M., see Rockstuhl, T.

Siegel, J.I., see Jeong, Y.

Siganos, A., Tabner, I.T., Capturing the role of societal affinity in cross-border mergers with the Eurovision Song Contest, 263

Singh, D., see Kumar, V.

Song, J., see Seo, E.

Steele, L.M., see Watts, L.L.

Steinke, C., see Dastmalchian, A.

$\mathrm{Su}, \mathrm{C} .-\mathrm{H}$. , see Allen, L.

$\mathrm{Su}, \mathrm{W} .$, see Tan, D.

Sugai, P., see Dastmalchian, A.

Tabner, I.T., see Siganos, A.

Tan, D., Su, W., Mahoney, J.T., Kor, Y., A review of research on the growth of multinational enterprises: A Penrosean lens, 498

Tang, N., see Dastmalchian, A.

Thang, T.T.N., see Dastmalchian, A.

Tian, A.W., see Backmann, J.

Tippmann, E., see Monaghan, S. 
Tsang, A., see Kanagaretnam, K.

Tsang, E.W.K., see Kano, L.

Ünal, M., see Gözübüyük, R.

Urtasun-Alonso, A., see Shijaku, E.

Useche, D., Miguelez, E., Lissoni, F., Highly skilled and well connected: Migrant inventors in crossborder M\&As, 737

Vahlne, J.-E., Johanson, J., The Uppsala model: Networks and micro-foundations, 4

van der Straaten, K., Pisani, N., Kolk, A., Unraveling the MNE wage premium, 1355

van Essen, M., see Kostova, T.

van Witteloostuijn, A., New-day statistical thinking: A bold proposal for a radical change in practices, 274

van Witteloostuijn, A., see Beugelsdijk, S.

Varnali, R., see Dastmalchian, A.

Verbeke, A., see Caligiuri, P.

Verbeke, A., see Fainshmidt, S.

Verbeke, A., see Lindner, T.

Verbeke, A., see Rosa, B.

Verbeke, A., The JIBS 2019 Decade Award: The Uppsala internationalization process model revisited: From liability of foreignness to liability of outsidership, 1

Villadsen, A.R., see Wulff, J.N.

Von Glinow, M.A., see Wang, S.L.

Wang, F., see Khurshed, A.

Wang, H., see Zhou, N.

Wang, S.L., Gu, Q., Von Glinow, M.A., Hirsch, P., Cultural industries in international business research: Progress and prospect, 665
Watts, L.L., Steele, L.M., Den Hartog, D.N., Uncertainty avoidance moderates the relationship between transformational leadership and innovation: A meta-analysis, 138

Welch, C., see Nielsen, B.B.

Westney, D.E., Rude awakening: threats to the global liberal order, 1195

Witt, M.A., see Fainshmidt, S.

$\mathrm{Wu}, \mathrm{F}$., see Huang, T.

$\mathrm{Wu}$, J., see Kim, H.

Wulff, J.N., Villadsen, A.R., Keeping it within bounds: Regression analysis of proportions in international business, 244

Xu, K., Hitt, M.A., Miller, S.R., The ownership structure contingency in the sequential international entry mode decision process: Family owners and institutional investors in familydominant versus family-influenced firms, 151

Yang, D., see Cannon, J.N. Yeung, H.W., see Kano, L. Yeung, H.W., see Kano, L. $\mathrm{Yu}, \mathrm{J}$., see Huang, T.

Zaheer, A., see Cuypers, I.R.P.

Zanfei, A., see Papanastassiou, M.

Zhang, B., see Huang, T.

Zhou, N., Wang, H., Foreign subsidiary CSR as a buffer against parent firm reputation risk, 1256

Zimmermann, A., see Ambos, T.C.

Zimmermann, A., see Caligiuri, P. 


\section{KEYWORD INDEX TO VOLUME 51}

absorptive capacity 23, 1541, 1579

adaptation 1557

adaptation on fitness landscapes 1541

alliances and joint ventures 1223

analysts following 1008

bargaining 829

born digitals 11

bundling model 1176

business 194

business-government interaction 1523

capital cities 1107

case theoretic approaches 326, 389, 906

catching up by hiring 1506

China 326, 1592

Chinese multinational enterprises 1076

cities 121

city-regions 1199

cognitive and emotional processes 4

collinearity 283

co-location 121

commitment 38

comparative institutional analysis 577

competitive advantage 1592

competitors 963

complexity 1557, 1623

configurational analysis 455

context-dependence in learning 1541

corporate governance 764

corporate political strategies 1592

corporate social responsibility 1034, 1256

corporate social responsibility reporting 389

corruption 414

cost asymmetry 1447

country-dyadic military conflicts 299

creativity 138

cross-border acquisitions (CBAs) 172, 299

cross-border investments 986, 1312

cross-border mergers and acquisitions 194,

263, 737

cross-border R\&D collaboration 851

cross-cultural management 353

cross-listing1008

cross-national distances 813

culture 1034

cultural gap bridging behaviors 1283

cultural industries 665 cultural intelligence 1283

cumulative abnormal return 299

Daniel Levinthal 1541

data access 887

Decade Award 4, 11, 23, 38

degree of internationalization 1133

delisting 1008

difference 1623

digital firm 11

digitization 11

(dis)economies of interdependence 1054

disparity 1623

disruption 1199

distance 1623

diversity 1623

divestment 1331

dividend repatriation 1312

domestic firms 1355

dynamism 1623

econometrics 244

economic freedom 1034

economic geography 50, 1523

Edith Penrose 498

emerging economies 23

emerging market firms 50, 1506

emerging markets 172, 665, 764

employees 1355

entry mode decisions 151

epistemology 1606

equity share 414

Eurovision Song Contest 263

event history analysis 414

evolution 4

exogenous shocks 1331

expansion speed 1107

expatriate 414

externalities 1415

family firms 151

family patents 963

fast-mover (dis)advantages 1076

financial contracting 95

financial performance 1076

firm heterogeneity 121

firm internationalization 1133

firm performance 764

firm value 72

foreign direct investment 1392

foreign divestment 788 
foreign sales to total sales (FSTS) 1133

foreign subsidiaries 1256, 1312

foreign subsidiaries in emerging markets 414

fractional regression 244

fsQCA 455

fuzzy sets 455

gender equality 1355

geographic distance 1107

geographic relational perspective 50

global cities 1199

global commodity chains 577

global factory 577

global firm 1133

global innovation 851, 1161

global production networks 577

global scope 1142

global strategy 1223

global value chains (GVCs) 432, 577, 1161, 1199

globalization 432

government 1592

greenfield investment 1506

group learning theory 851

growth 498

headquarters-subsidiary roles and relations 906, 1256

hierarchical modeling 283

high-performance work systems 353

history of FDI 1392

home regionalization 1076

hybrid organization 906

IB paradigm 1483

innovation 138, 623, 665, 963, 1415, 1557

innovation capabilities 1506

input-output tables 432

insider information 218

institutions 1415

institutional context 467

institutional distance 467

institutional environment 764

institutional investors 151

institutional ownership1008

institutional theory 23, 389, 467, 1223

integration and responsiveness 906

integration-responsiveness 414

interfirm influence 1331

intergroup relations 299

inter-location ties 714

internalization theory 1592 international 194

international business 498

international business research 1623

international diversification 1133

international mutual fund 218

international patenting 963

international relations 299

international scope 1133

international strategic alliances 813

international taxation 1415

internationalization 11, 38, 50, 172, 665, 1107

internationalization speed 1076

interorganizational relationships 1161

interorganizational ties 714

inter-personal ties 714

inventive performance 1054

inventors 737

investing 218

investor protection 72

knowledge 963

knowledge accumulation 887

knowledge and productivity spillovers 1392

knowledge bases 121

knowledge clusters 963

knowledge strategies 1592

knowledge transfer 1199

knowledge transfer and innovation 1523

labor market regulations 194

labor standards 194

latecomer companies 1506

learning 23

legal systems 1415

liability of foreignness 95, 1223

liability of outsidership 23

liability/liabilities of foreignness

literature review 714

loan enforcement 95

location choice 121

longitudinal analysis 326, 813

managerial myopia 1447

mandatory IFRS adoption 1008

market discipline 1447

marketing strategy 38

meta-analysis 138, 933

methodological bandwidth 1483

methodological innovation 1483

methodological rigor 1483

methodological trends 1483

methodology 1606, 1623 
methods complexity 1483

methods diversity 1483

micro-foundations 389

migration 737

MNE geography 1199

MNE strategy 1199

MNE-host country relations 829

MNE-SME cooperation 1161, 1176

multicollinearity 283

multi-culturals 1283

multi-level analysis 764, 1355

multimarket competition 963

multinational corporations (MNCs) and enterprises

(MNEs) 121, 194, 389, 432, 498, 623, 714, 788, 851, 963, 1107, 1133, 1142, 1331, 1355, $1392,1415,1557$

multinational enterprise (MNE) subsidiaries 538

multi-party cooperation 1523

multiple regression analysis 95, 244, 1223, 1283

multiplexity 1623

multiplicity 1623

national culture 933

network 4

network analysis 714, 829

network centrality 813

network effects 11

network theory 714, 764, 788

networks 23, 38, 764, 1176

new internalization theory 1392

null hypothesis testing 274

off shoring 1199

open science 887

organizational aspirations 813

organizational forms 1415

organizational learning 38, 121, 986, 1557

organizational paradox 906

organizational performance 353

organizational support 933

outward foreign direct investment (OFDI) 50

paradox of control 1392

PCT patents 737

performance 38

pharmaceutical industry 851 political risk 829

process model 326

production 1142

proportional dependent variable 244

qualitative comparative analysis 455

quantitative research methods 283

questionable research practices 274

R\&D 121, 623

$\mathrm{R} \& \mathrm{D}$ activities 1054

R\&D internationalization 1506

radical change 4

random parameter models 121

ratio of international market shares (RIMS) 1133

regional scope 1142

regionalization 1054

regression analysis 283

reputation risk spillover 1256

research design 1606

research methods 887, 1606, 1623

resource adjustments 1447

resource dependence theory 23

review 538

rivals 963

sales 1142

scalability 11

sequential decision making 151

short-termism 1447

small- and medium-sized enterprises 326

SME internationalization 1161

social inclusion 665

social influence 829

social networks 714

social optimality 1415

social-commercial tensions 906

social-exchange theory 933

socially enabling mechanisms 1579

societal affinity 263

societal culture 353

speed 172

staffing 414

statistical significance 274

stock liquidity 72

strategic knowledge capabilities 38 
subsidiary evolution 538 subsidiary innovation 538 subsidiary management 538 subsidiary managers 389 subsidiary networks 1223 subsidiary portfolio 1107 subsidiary strategy 538 subsidiary survival and market exit 414 subsidiary-HQ relationships 714 survey methods 1283 Sustainable Development Goals 1161 syndicate 95 syndicated bank loans 218 syndication 986

takeover laws 1447 tax avoidance 1312 team composition 851 teams and teamwork 1283 temporal consistency 1331 territorial tax system 1312 terrorism 1331 the organizational perspective of corruption 414 theory advancement 1606 theory of FDI and the MNE 1523 theory progress 1606

Tobit 244

trade in value-added 432

tranche 95

transaction cost theory 1176

transfer of practices 389

transformational leadership 138

translation 389

transparency 829,887

trial and error experiments 1579

triangulation 1483

trust 326

trust erosion 326

ultimate owner 1392

uncertainty 414, 788

uncertainty avoidance 138

value chain 1176

value creation 299

venture capital 986

wage premia 1355

worldwide tax system 1312

Publisher's Note Springer Nature remains neutral with regard to jurisdictional claims in published maps and institutional affiliations. 